\title{
MutT Homolog-1 Attenuates Oxidative DNA Damage and Delays Photoreceptor Cell Death in Inherited Retinal Degeneration
}

\author{
Yusuke Murakami, ${ }^{,}$Yasuhiro Ikeda, ${ }^{* \dagger}$ \\ Noriko Yoshida, ${ }^{*}$ Shoji Notomi, ${ }^{*}$ Toshio Hisatomi, ${ }^{*}$ \\ Sugako Oka, ${ }^{\dagger \ddagger}$ Gabriele De Luca, ${ }^{\S}$ \\ Yoshikazu Yonemitsu, ${ }^{\text {" }}$ Margherita Bignami, ${ }^{\S}$ \\ Yusaku Nakabeppu, ${ }^{\dagger \ddagger}$ and Tatsuro Ishibashi ${ }^{\star}$ \\ From the Department of Ophthalmology,* Graduate School of \\ Medical Sciences, the Research Center for Nucleotide Pool, ${ }^{\dagger}$ the \\ Division of Neurofunctional Genomics, ${ }^{\ddagger}$ Medical Institute of \\ Bioregulation, and the RED Laboratory for Innovative \\ Biotherapeutics, "Graduate School of Pharmaceutical Sciences, \\ Kyushu University, Fukuoka, Japan; and the Experimental \\ Carcinogenesis Division, ${ }^{\S}$ Department of Environment and \\ Primary Prevention, Istituto Superiore di Sanita, Rome, Italy
}

Retinitis pigmentosa (RP) is a genetically heterogenous group of inherited retinal degenerative diseases resulting from photoreceptor cell death and affecting $>1$ million persons globally. Although oxidative stress has been implicated in the pathogenesis of RP, the mechanisms by which oxidative stress mediates photoreceptor cell death are largely unknown. Here, we show that oxidation of nucleic acids is a key component in the initiation of death-signaling pathways in $\mathrm{rd10}$ mice, a model of RP. Accumulation of 8-oxo-7,8-dihydro-2' deoxyguanosine (8-oxo-dG) increased in photoreceptor cells, and especially within their nuclei, in $\mathrm{rd10}$ mice as well as in Royal College of Surgeons rats, another model of RP caused by different genetic mutations. Vitreous samples from humans with RP contained higher levels of 8-oxo-dG excreted than samples from nondegenerative controls. Transgenic overexpression of human MutT homolog-1, which hydrolyzes oxidized purine nucleoside triphosphates in the nucleotide pool, significantly attenuated 8-oxo-dG accumulation in nuclear DNA and photoreceptor cell death in rd10 mice, in addition to suppressing DNA single-strand break formation, poly(ADP-ribose) polymerase activation, and nuclear translocation of apoptosis-inducing factor. These findings indicate that oxidative DNA damage is an important process for the triggering of photoreceptor cell death in $\mathrm{rd10}$ mice and suggest that stimulation of DNA repair enzymes may be a novel therapeutic approach to attenuate photoreceptor cell loss in RP. (Am J Pathol 2012, 181:1378-1386; http://dx. doi.org/10.1016/j.ajpath.2012.06.026)

Retinitis pigmentosa (RP) is a group of inherited retinal degenerative diseases resulting from rod and cone photoreceptor cell death and affecting 1.5 million people globally. Molecular genetic analyses have identified $>45$ genetic mutations in patients with $\mathrm{RP}^{1}$; however, the mechanisms by which these mutations cause photoreceptor cell death are largely unknown and these diseases remain intractable.

Oxidative stress has been implicated in the pathogenesis of neurodegenerative disorders, including retinal degeneration. Retinal oxidative damage increases in animal models of RP and antioxidant treatment suppresses rod and cone photoreceptor cell death in these animals, ${ }^{2,3}$ indicating the deleterious effects of oxidative damage to photoreceptor cells. By contrast, oxidative stress is also involved in cell survival signaling. ${ }^{4}$ For example, hydrogen peroxide generation in response to cellular stress activates the Akt pathway and mediates the survival of retina-derived cells. ${ }^{5}$ Given the diverse functions of oxidative stress, identification of the processes by which oxidative stress induces photoreceptor cell death is critical to developing new treatments for retinal and neurodegenerative diseases.

Reactive oxygen species affect cellular functions by oxidizing lipids, proteins, and nucleic acids. Excessive

\footnotetext{
Supported by the Japanese Ministry of Education, Culture, Sports, Science, and Technology (grants-in-aid 20791259 to Y.I. and 22221004 to Y.N.).

Accepted for publication June 18, 2012.

Y.M. and Y.I. contributed equally to this work.

Supplemental material for this article can be found at $h t t p: / / a j p$. amjpathol.org or at $h t t p: / / d x . d o i . o r g / 10.1016 / j$. ajpath.2012.06.026.

Address reprint requests to Yasuhiro Ikeda, M.D., Ph.D., Department of Ophthalmology, Graduate School of Medical Sciences, or Yusaku Nakabeppu, D.Sc., D.V.M., Division of Neurofunctional Genomics, Medical Institute of Bioregulation, Kyushu University, 3-1-1 Maidashi, Higashi-ku, Fukuoka, 812-8582, Japan. E-mail: ymocl@pathol1.med.kyushu-u.ac.jp or yusaku@bioreg.kyushu-u.ac.jp.
} 
oxidation of DNA leads to the formation of abasic (apurinic/apyrimidinic) sites and single-strand breaks (SSBs) and initiates death-signaling pathways. ${ }^{6,7}$ Accumulation of oxidative DNA damage is associated with various neurodegenerative diseases, such as Parkinson's disease and Alzheimer's disease. ${ }^{8,9}$ Mammalian cells have multiple repair mechanisms against oxidative damage to nucleic acids. 8-Oxo-7,8-dihydroguanine (8OxOG) DNA glycosylase-1 excises oxidized guanine from DNA. ${ }^{10}$ Alternatively, the MutT homolog-1 (MTH1) hydrolyzes oxidized purine nucleoside triphosphates to the monophosphate form, thereby avoiding incorporation of oxidized nucleotides into DNA. ${ }^{11,12}$ We previously reported that $M$ th1 deficiency increases mitochondrial oxidative DNA damage and cell death in dopamine neurons after 1-methyl-4-phenyl-1,2,3,6-tetrahydropyridine administration. ${ }^{13}$ Conversely, transgenic overexpression of human MTH1 (hMTH1) protects against the striatal degeneration induced by 3-nitropropionic acid. ${ }^{14}$ These findings suggest that oxidative DNA damage may play a role in the pathogenesis of neurodegeneration. However, little is known about the contribution of DNA oxidation to the photoreceptor cell death caused by genetic factors. Furthermore, it remains unclear which kind of oxidative DNA damage - mitochondrial or nuclear DNA damage or both-accumulates and how it causes photoreceptor cell death in inherited retinal degeneration.

In the present study, we show that DNA oxidation was increased in an early phase of retinal degeneration in two different models of RP and in the eyes of patients with RP. Transgenic overexpression of hMTH1 in a mouse RP model attenuated accumulation of oxidative DNA damage, prevented the subsequent poly(ADP-ribose) polymerase (PARP) activation, and delayed photoreceptor cell death. These findings implicate oxidative DNA damage as an important component in the pathogenesis of RP.

\section{Materials and Methods}

\section{Animals}

All animal experiments adhered to the statement of the Association for Research in Vision and Ophthalmology, and protocols were approved by the Committee for Animals, Recombinant DNA, and Infectious Pathogens Experiments at Kyushu University. B6.CXB1-Pde6 $\beta^{\text {rd10 } / J ~}$ (rd10) and age-matched wild-type (WT) C57BL/6 mice were purchased from The Jackson Laboratories (Bar Harbor, ME). Adult Royal College of Surgeons (RCS) rats and age-matched SD rats were maintained humanely. hMTH1 transgenic (hMTH1-Tg) mice were generated as described previously ${ }^{14}$ and backcrossed to the C57BL/6 background. Rd10 mice were crossed with hMTH1-Tg mice, and the littermates from rd10; $\mathrm{hMTH1- \textrm {Tg } ^ { + / - } \text { males }}$ and rd10; hMTH1- $\mathrm{Tg}^{+/-}$females were used for the experiments. Rd10 mutation in exon 13 of the $P d e 6 \beta$ gene [CGC (Arg) $\rightarrow$ TGC (Cys)] was identified by PCR with the use of the forward primer 5'-TCTCAGAACCCACATGTACT-3' and reverse primer 5'-TGATTCATCTAGCCCATCC-3' and subsequent direct sequencing. The
hMTH1 transgene was analyzed by PCR with the use of previously described primers. ${ }^{14}$ Heterozygous and homozygous $h M T H 1-T g$ mice were distinguished by real-time PCR with the use of primers for hMTH1 (forward primer, 5'-TTGAGTTCGTGGGCGAGC-3'; reverse primer, 5'GCATGGGCGCATTTCG-3'; and MGB probe, 5'-FAM-CACAGACAGCATCCAG-MGB-3') and for RNase P (forward primer, 5'-GCCGGAGCTTGGAACAGA-3'; reverse primer, 5'-GGTGCCTCACCTCAGCCAT-3'; TaqMan probe, 5' -VICCACGGCCAGCAGTGCGAGTTCA-TAMRA-3'; Applied Biosystems, Foster City, CA).

\section{8-oxo-dG Immunostaining}

Immunostaining for 8-oxo-dG in nuclear and mitochondrial DNA was performed as previously described..$^{13}$ Deparaffinized sections were pretreated with $5 \mathrm{mg} / \mathrm{mL}$ RNase (Sigma-Aldrich, St. Louis, MO) at $37^{\circ} \mathrm{C}$ for 1 hour to eliminate cellular RNA. To increase the sensitivity of 8-oxo-dG in nuclear DNA, the sections were further treated with $2 \mathrm{~N} \mathrm{HCl}$ at room temperature for 10 minutes, and then washed with Tris- $\mathrm{HCl}, \mathrm{pH} 7.5$, for 10 minutes. Samples pretreated with RNase or RNase $+\mathrm{HCl}$ were incubated with N45.1 anti-8oxo-dG antibody [1:100; Japan International Cooperation Agency (JAICA), Fukuroi, Japan] at $4^{\circ} \mathrm{C}$ overnight. Then signals were developed with the avidin-biotinylated peroxidase complex method (Dako, Carpinteria, CA). A nonimmune serum was used as a negative control.

\section{Patients}

A total of 20 eyes with epiretinal membrane (ERM; idiopathic: 13 eyes of 12 patients; secondary to RP: 7 eyes of 6 patients, respectively) underwent a pars plana vitrectomy at Kyushu University Hospital (Fukuoka, Japan), and the vitreous fluid was collected. The investigation was performed with approval from the institutional review board and was conducted in accordance with the tenets of the Declaration of Helsinki on biomedical research involving human subjects. The details on patients with RP are given (see Supplemental Table S1 at http://ajp. amjpathol.org). All six patients with RP developed rodcone dystrophy bilaterally, with a variable extent of visual restriction. The visual fields were evaluated with Goldmann kinetic perimetry, and the vertical diameter of the visual field for the 1/4 target was measured as previously described..$^{15}$ No patient had any associated hearing loss or other systemic diseases. The mode of inheritance was autosomal dominant in one patient, autosomal recessive in two patients, and spontaneous (an individual patient with no family history of RP) in three patients. The frequency of the type of inheritance was consistent with a previous study of Japanese patients with RP (16.9\% dominant, $25.2 \%$ recessive, $1.6 \%$ X-linked, and $56.3 \%$ spontaneous). ${ }^{16}$ Genetic tests were not performed.

\section{ELISA}

Total DNA in the mouse and rat retinas was extracted with a DNeasy Blood and Tissue Kit (QIAGEN, Valencia, CA). At all of the stages of manipulation $10 \mathrm{mmol} / \mathrm{L} \mathrm{2,2,6,6-}$ 
tetramethylpiperidine-N-oxyl (Wako, Osaka, Japan) was added, as previously described. ${ }^{17}$ The DNA samples were digested with 5 units of Nuclease P1 (Sigma-Aldrich) at $37^{\circ} \mathrm{C}$ for 1 hour in $20 \mathrm{mmol} / \mathrm{L}$ sodium acetate, $\mathrm{pH}$ 5.2, followed by treatment with 10 units of alkaline phosphatase (Sigma-Aldrich) at $37^{\circ} \mathrm{C}$ for 1 hour in $100 \mathrm{mmol} / \mathrm{L}$ Tris, $\mathrm{pH}$ 7.5. The reaction mixture was centrifuged for 5 minutes at 10,000 $\times g$, and the supernatant fluid was used for 8-oxo-dG enzyme-linked immunosorbent assay (ELISA) (Cell Biolabs, San Diego, CA) according to the instructions of the manufacturer. Briefly, $1 \mu \mathrm{g}$ of digested DNA samples or 8-oxo-dG standards was incubated with anti-8-oxo-dG antibody in wells precoated with 8-oxo-dG. The antibodies that bound to 8-oxo-dG in the digested DNA samples were washed away, and the antibodies that bound to 8-oxo-dG on the plate were quantified with enzyme-labeled secondary antibody. The 8-oxo-dG concentration obtained from a standard curve was converted to the 8-oxo-dG amount in $1 \mu \mathrm{g}$ total DNA with the following calculation: [8-oxo-dG concentration $(\mathrm{pg} / \mu \mathrm{L}) \times 50$ $\mu \mathrm{L}] / 1 \mu \mathrm{g}$ total DNA. Vitreous samples were centrifuged at $10,000 \times g$ for 5 minutes, and the supernatant fluids were subjected to 8-oxo-dG ELISA assay as described earlier.

\section{Histologic Examination}

The mouse eyes were enucleated, fixed with $4 \%$ paraformaldehyde in PBS for 24 hours, and then mounted in paraffin. Sections $5 \mu \mathrm{m}$ thick were prepared along the horizontal meridian. The sections were subsequently stained with H\&E. Five sections were randomly selected from each eye. The number of cells in the outer nuclear layer (ONL) was counted in a 200 $\mu \mathrm{m} \times 200 \mu \mathrm{m}$ square area in the central (400 $\mu \mathrm{m}$ from the optic nerve), mid-peripheral (800 $\mu \mathrm{m}$ from the optic nerve), and peripheral (1200 $\mu \mathrm{m}$ from the optic nerve) regions of the retina in the nasal hemisphere. The tissue samples were assigned numbers and letters, and the conditions were masked from the observers.

\section{TUNEL Staining}

The TUNEL procedure and quantification of TUNEL-positive cells were performed with an ApopTag Fluorescein Direct In Situ Apoptosis Detection Kit (Chemicon International, Temecula, CA) according to the instructions of the manufacturer. The mid-peripheral retina was photographed, and the number of TUNEL-positive cells in the ONL was counted by masked observers. The retinal area was measured by Image $\mathrm{J}$ software version $1.43 \mathrm{u}(\mathrm{Na}-$ tional Institutes of Health, Bethesda, MD). The data are expressed as TUNEL-positive cells $/ \mathrm{mm}^{2}$ of retinal area.

\section{Immunofluorescence}

Immunofluorescence was performed as previously reported. ${ }^{18}$ Paraffinized sections were used for single-strand DNA and poly(ADP-ribose) (PAR) staining, and cryosections were used for apoptosis-inducing factor (AIF) and voltage-dependent anion-selective channel (VDAC) staining. Samples were incubated with anti-single strand DNA
(1:200; Dako), anti-PAR (1:200; Trevigen, Gaithersburg, MD), anti-AIF (1:100; Cell Signaling, Danvers, MA), or antiVDAC (1:100; Cell Signaling) at $4^{\circ} \mathrm{C}$ overnight. A nonimmune serum was used as a negative control. Alexa Fluor 488- or Alexa Fluor 594-conjugated antibodies (Invitrogen, Carlsbad, CA) was used as secondary antibody. Immunofluorescent images were acquired with an Olympus BX51 microscope (Olympus, Tokyo, Japan) with a fluorescence attachment or Leica confocal microscope (Leica, Wetzlar, Germany). Double-staining pictures were taken with Leica confocal microscope.

\section{Western Blot Analysis}

The neural retina was collected at the indicated time points. Protein $(30 \mu \mathrm{g})$ per sample was separated on SDS-PAGE and transferred onto a polyvinylidene difluoride membrane. After blocking with 3\% nonfat dried milk, the membrane was reacted with anti-PAR (1:3000; Trevigen). Immunoreactivity for PAR was visualized with the ECL Plus detection reagents (Amersham Biosciences, Buckinghamshire, United Kingdom). Lane-loading differences were normalized by $\beta$-actin (1:1000; Cell Signaling).

\section{Calpain Activity}

Calpain activity was measured with a Calpain Activity Assay kit (Biovision, Mountain View, CA) according to the instructions of the manufacturer. The calpain substrate (Ac-LLY-AFC) was incubated with retinal lysates (200 $\mu \mathrm{g}$ of protein) for 60 minutes at $37^{\circ} \mathrm{C}$ in the dark.

\section{PARP and Calpain Inhibitor Treatment}

3,4-Dihydro-5-[4-(1-piperidinyl)butoxy]-1(2H)-isoquinolinone (DPQ; PARP inhibitor) and MDL28170 (calpain inhibitor) were purchased from Enzo Lifesciences (Plymouth Meeting, PA). Rd10 mice received once daily intraperitoneal injection of DPQ $(20 \mu \mathrm{g} / \mathrm{g})$, MDL28170 $(25 \mu \mathrm{g} / \mathrm{g})$, or vehicle (1\% dimethyl sulfoxide in PBS) from p19 to p26.

\section{Statistical Analysis}

All values were expressed as the mean \pm SEM. Statistical differences between two groups were analyzed by MannWhitney U-test. Multiple group comparison was performed by analysis of variance followed by Tukey-Kramer adjustments. Differences were considered significant at $P<0.05$.

\section{Results}

\section{Oxidative DNA Damage in Animal Models and Patients with RP}

Rd10 mice are an animal model of RP harboring homozygous mutation in the $\beta$-subunit of the $P d e 6 \beta$ gene that develop progressive retinal degeneration with onset around p18. ${ }^{19}$ The course of retinal degeneration in $r d 10$ mice is slower than that of $r d 1$ mice, another Pde6 $\beta$ mutant mouse model, and it has been suggested that 

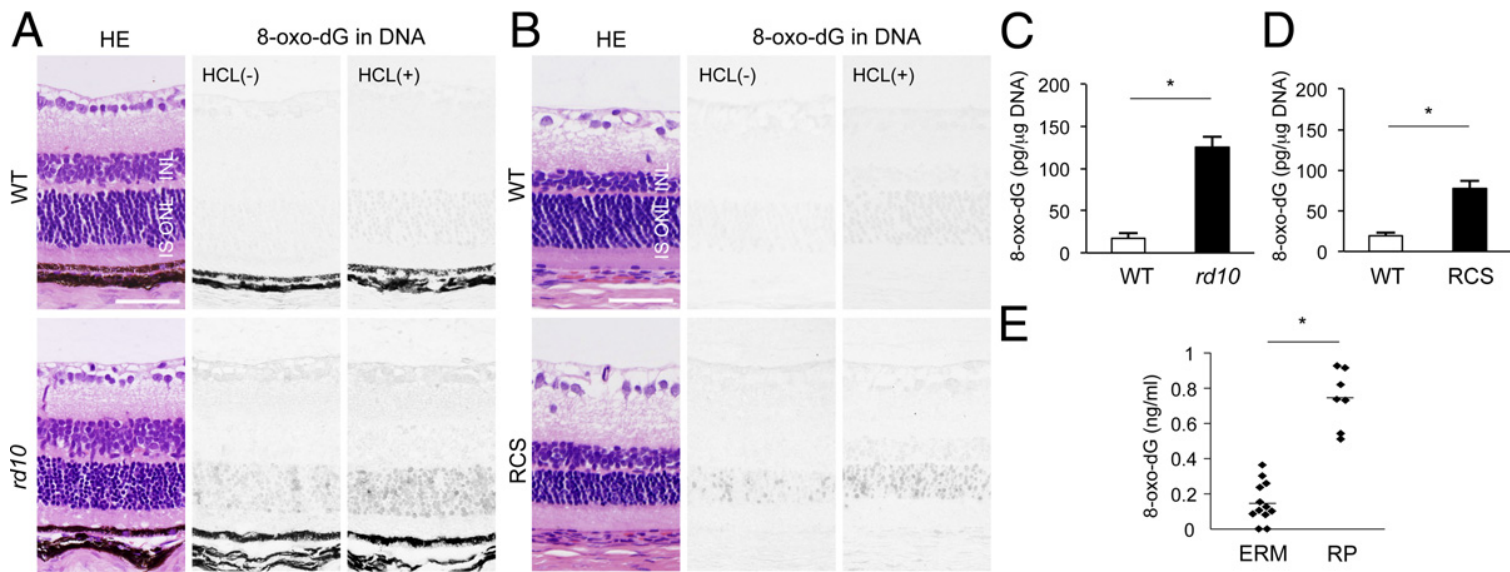

Figure 1. Accumulation of oxidative DNA damage in $r d 10$ mice, RCS rats, and human patients with RP. A and B: H\&E staining and immunohistochemical detection of 8-oxo-dG in the retina of p21 rd10 mice (A) and of p28 RCS rats (B). The sections were pretreated with RNase before incubation with N45.1 antibody to detect 8-oxo-dG in DNA. HCl pretreatment was used for denaturing the nuclear DNA, thereby enhancing the detection of 8-oxo-dG in nuclear DNA. Scale bar $=50 \mu \mathrm{m}$. INL: inner nuclear layer. C: ELISA for 8-oxo-dG in total DNA prepared from the retina of p21 $r d 10$ and WT mice $(n=6$ each $) .{ }^{*} P=0.0039$. D: ELISA for 8-oxo-dG in total DNA prepared from the retina of p28 RCS and Sprague-Dawley rats $(n=7$ and 8$)$. ${ }^{*} P=0.0012$. E: Concentrations of 8 -oxo-dG in the vitreous fluid were measured by ELISA (idiopathic ERM, $n=13$; and ERM secondary to RP, $n=7$ ). Vitreous 8 -oxo-dG levels in patients with RP were significantly higher than levels in nondegenerative controls (ERM). ${ }^{*} P=0.0003$.

rd10 mice might be more analogous to later onset forms of RP. ${ }^{20}$ To assess the levels and distribution of oxidative DNA damage in retinal degeneration, we performed immunohistochemistry for 8-oxo-dG, a major oxidative lesion in DNA or nucleotides. ${ }^{21}$ We previously described that the N45.1 monoclonal antibody preferentially detects 8-oxo-dG in mitochondrial DNA in samples pretreated with RNase, whereas it reacts with 8-oxo-dG in nuclear DNA in samples pretreated with RNase and $\mathrm{HCl}^{13}$ Thus, we applied these two different pretreatments for the retinal sections. Among samples pretreated with RNase alone, the p21 rd10 retina sections showed stronger immunoreactivity for 8-oxo-dG in the photoreceptor cell layer compared with the WT retina sections. Unexpectedly, however, the signal was mainly localized in the ONL but not in the inner segment (IS), a mitochondria-rich portion of photoreceptor cells (Figure 1A). Double immunostaining with 8-oxo-dG and a mitochondrial marker, VDAC, showed that most of the 8-oxo-dG staining was not colocalized with VDAC (see Supplemental Figure S1 at http://ajp.amjpathol.org), suggesting that the photoreceptor nuclear DNA in rd10 mice may be strongly oxidized and that the N45.1 antibody may detect the strong 8-oxo-dG accumulation in the photoreceptor nuclei even without $\mathrm{HCl}$ pretreatment. In accordance with this idea, rd10 retina samples pretreated with $\mathrm{RNase}$ and $\mathrm{HCl}$ exhibited intense 8-oxo-dG immunoreactivity in photoreceptor nuclei (Figure 1A). In contrast, the WT retina showed minimal immunoreactivity even with $\mathrm{HCl}$ pretreatment (Figure 1A). No positive staining was found by nonimmune mouse IgG in samples with either pretreatment (data not shown).

We next assessed oxidative DNA damage in RCS rats, another clinically relevant model of RP caused by a Mertk mutation. ${ }^{22,23}$ The retina of p28 RCS rats showed strong immunoreactivity for 8-oxo-dG in the $\mathrm{ONL}$, which was further enhanced by $\mathrm{HCl}$ pretreatment (Figure 1B). In contrast, the normal rat retina sections used as controls showed minimal staining for 8-oxo-dG with either pre- treatment (Figure 1B). Quantitative measurement of 8-oxo-dG by ELISA confirmed that the amounts of 8-oxo-dG in total DNA prepared from the retina of $r d 10$ mice and RCS rats were significantly higher than those in the controls (Figure 1, C and D).

8-Oxo-dG content excreted in the urine, serum, or cerebrospinal fluid has been shown to serve as a biomarker of oxidative damage ${ }^{24}$ and to be increased in patients with Parkinson's disease or acute encephalopathy. 25,26 Therefore, we next examined the levels of 8-oxo-dG excretion in the vitreous samples from human patients with $\mathrm{RP}$ and nondegenerative 'controls (idiopathic ERM). The vitreous levels of 8-oxo-dG in patients with ERM secondary to RP $(0.75 \pm 0.06 \mathrm{ng} / \mathrm{mL})$ were significantly higher than levels in patients with ERM alone (Figure 1E).

\section{hMTH1 Overexpression Delays Photoreceptor Cell Death in a Mouse Model of RP}

To assess the role of oxidative DNA damage in inherited retinal degeneration, we crossed $r d 10$ mice with transgenic mice expressing hMTH1 cDNA. ${ }^{14}$ The number of photoreceptor cells was counted in the peripheral, midperipheral, and central regions of the retina at p26. The results showed that there was a 1.5-fold increase in the number of photoreceptor cells at the mid-peripheral retina of rd10 mice with a hemizygous hMTH1 transgene (rd10; hMTH1- $\mathrm{Tg}^{+/-}$) compared with control rd10 mice (rd10; $\mathrm{hMTH1}^{-\mathrm{Tg}^{-/-}}{ }^{-}$(Figure 2, A and B). Rd10 mice with a homozygous hMTH1 transgene (rd10; hMTH1- $\mathrm{Tg}^{+/+}$) exhibited significant protection of photoreceptor cells at the mid-peripheral and peripheral retina (Figure 2, A and $B$ ) and retained 1.8-fold higher number of photoreceptor cells than control rd10 mice at the mid-peripheral retina. In contrast, the retinal structure in WT mice was not affected by the homozygous hMTH1 transgene (see Supplemental Figure S2 at $h$ ttp://ajp.amjpathol.org). Because the mid-peripheral region showed the greatest prevention of photore- 
A
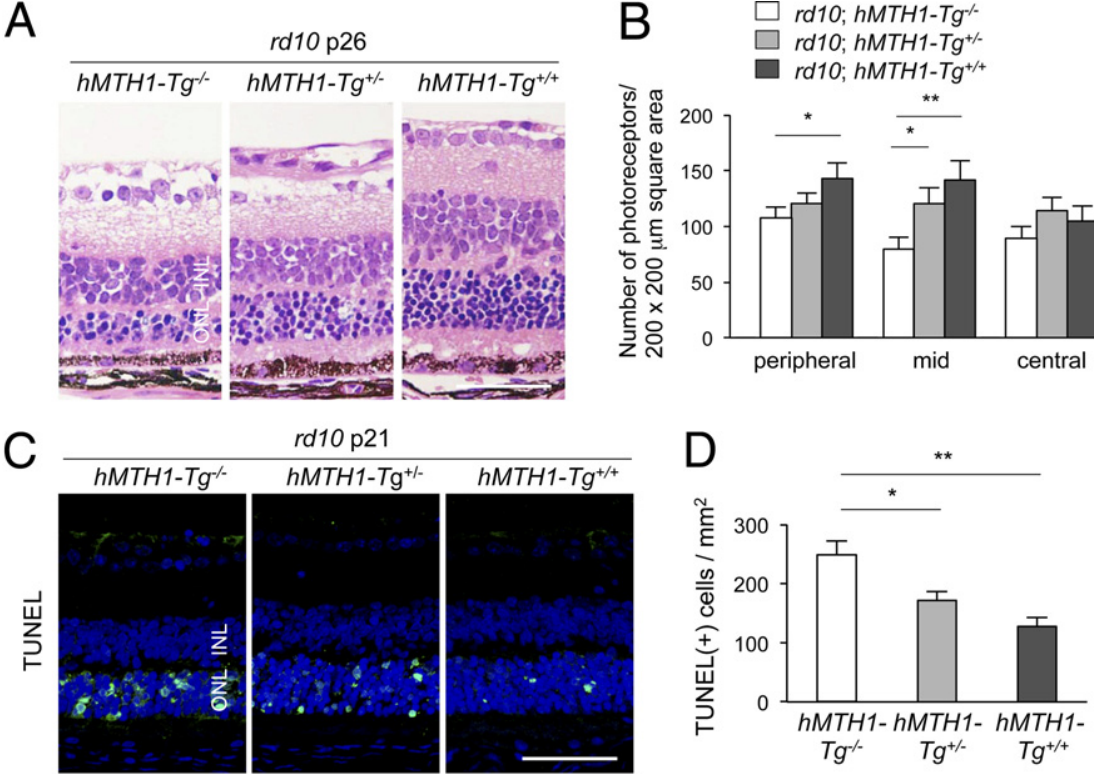

E
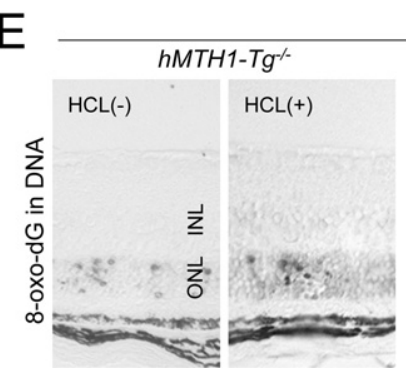

$\mathrm{B} \quad \square$ rd10; hMTH1-Tg/
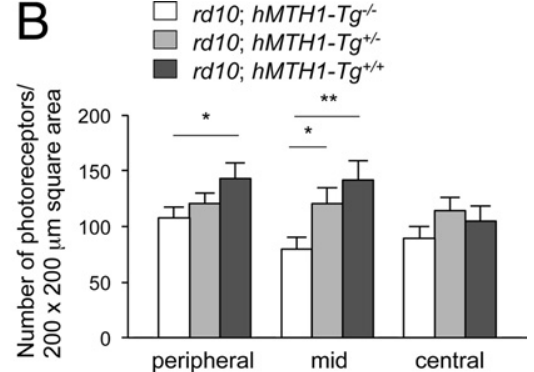

peripheral

mid $r d 10 \mathrm{p} 21$ hMTH1-Tg+/-

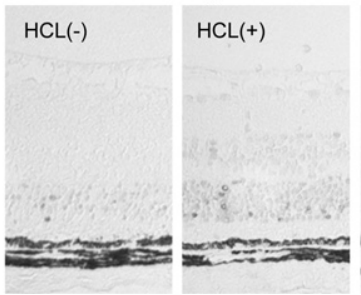

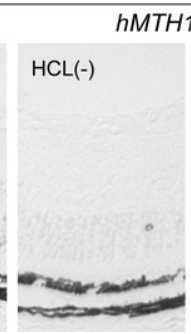

Figure 2. Transgenic hMTH1 expression attenuates oxidative DNA damage and delays photoreceptor cell death in $r d 10$ mice. A: Histologic findings of the mid-peripheral retina in p26 rd10 mice without hMTH1 expression ( $h M T H 1-\mathrm{Tg}^{-1-}$ ), with hemizygous hMTH1 expression ( $\left.h M T H 1-\mathrm{Tg}^{+-}\right)$, or with homozygous hMTH1 expression ( $b M T H 1$ $\left.\mathrm{Tg}^{+/+}\right)$. INL, inner nuclear layer. B: Quantitative analysis of cells in the outer nuclear layer (ONL). The number of nuclei in the ONL in a $200 \mu \mathrm{m} \times$ $200 \mu \mathrm{m}$ square area was counted at the peripheral, mid-peripheral, and central regions of the retina ( $h$ MTH1- $\mathrm{Tg}^{-1-}, n=12 ;$ hMTH1- $\mathrm{Tg}^{+/-}, n=18$ bMTH1- $\mathrm{Tg}^{+/+}, n=12$ ). Transgenic hMTH1 overexpression significantly suppressed photoreceptor

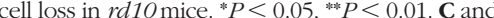
D: TUNEL (green) and DAPI (blue) staining $(\mathbf{C})$ and quantification of TUNEL-positive photoreceptor cells $(\mathbf{D})$ in the retina of $r d 10$ mice. Transgenic expression of hMTH1 substantially reduced the number of TUNEL-positive cells in the ONL ( $h M T H 1-\mathrm{Tg}^{-/-}, n=5 ;$ bMTH1- $\mathrm{Tg}^{+/-}, n=9$; hMTH1-Tg $\left.{ }^{+/+}, n=6\right) .{ }^{*} P<0.05,{ }^{* * *} P<0.01 . \mathbf{E}$ : Immunohistochemical detection of 8 -oxo-dG in the retina of $r d 10$ mice. The hemizygous hMTH1 transgene decreased 8-oxo-dG accumulation in photoreceptor cells, and this decrease was aug mented by the homozygous hMTH1 transgene. Scale bars: $50 \mu \mathrm{m}(\mathbf{A}, \mathbf{C}$, and $\mathbf{E})$. ceptor cell loss by hMTH1 overexpression in rd10 mice, we assessed this region of the retina in the following experiments. TUNEL staining confirmed that TUNEL-positive cells in the ONL were substantially reduced in rd10;hMTH1$\mathrm{Tg}^{+/-}$and rd10; $\mathrm{hMTH1}-\mathrm{Tg}^{+/+}$mice compared with TUNELpositive cells in rd10; $\mathrm{hMTH1}-\mathrm{Tg}^{-1-}$ mice (Figure 2, C and D). Furthermore, immunohistochemistry for 8-oxo-dG showed that the hemizygous hMTH1 transgene decreased 8-oxo-dG accumulation in photoreceptor nuclei, and this decrease was augmented by the homozygous hMTH1 transgene (Figure 2E). These data indicate that oxidative DNA damage in photoreceptor nuclei contributes to the photoreceptor cell death in rd10 mice.

\section{Formation of Nuclear SSBS and PARP Activation in rd10 Mice}

We recently described that accumulation of oxidative DNA damage results in the formation of SSBs in nuclear and mitochondrial DNA in mouse embryonic fibroblasts. ${ }^{27}$ In addition, we reported that DNA oxidation in the nucleus and mitochondria independently triggers two distinct cell death pathways: nuclear DNA oxidation mediates PARP activation and mitochondrial DNA oxidation activates calpain. Thus, we next examined the involvement of these pathways during retinal degeneration. Immunofluorescence for single-strand DNA showed intense staining in photoreceptor nuclei of rd10 mice but not of
WT mice at p21 (Figure 3A). We also performed immunofluorescence for PAR polymer, because this polymer is known to be produced on PARP activation. The results showed intense staining for PAR in the ONL of rd10 mice (Figure 3B). In contrast, the WT retina showed weak staining for PAR in the ONL (Figure 3B). Western blot analysis for PAR found that the rd10 retina contained poly(ADP)-ribosylated proteins of $\sim 110 \mathrm{kDa}$ and $\sim 170 \mathrm{kDa}$, whereas the WT retina contained mainly the $\sim 110-\mathrm{kDa}$ protein (Figure $3 \mathrm{C}$ ). This higher band of PAR-protein is reported to be produced during PARP-dependent cell death. ${ }^{28}$ Densitometric analysis showed that the amount of the $\sim 170-\mathrm{kDa}$ PAR-protein was increased 28 -fold in the rd10 retina compared with the WT retina (Figure 3D), suggesting that the PARP pathway is activated in rd10 mice. However, calpain activity in retinal extracts increased 1.3-fold in the rd10 retina compared with the WT retina (Figure 3E).

Activation of the PARP and calpain pathways has been reported in animal models of $\mathrm{RP}^{29-31}$; however, their contribution to photoreceptor cell loss in vivo remains unknown. To investigate the role of PARP and calpain in retinal degeneration, we treated rd10 mice with a PARP inhibitor and calpain inhibitor. Rd10 mice received daily intraperitoneal injection of a PARP inhibitor, DPQ $20 \mu \mathrm{g} / \mathrm{g}$, and/or a calpain inhibitor, MDL28170 (25 $\mu \mathrm{g} / \mathrm{g})$, from p19 to p26. The doses of the compounds were selected according to previous reports that showed protective effects on ischemic brain injury. ${ }^{32,33}$ Treatment with the PARP and calpain 

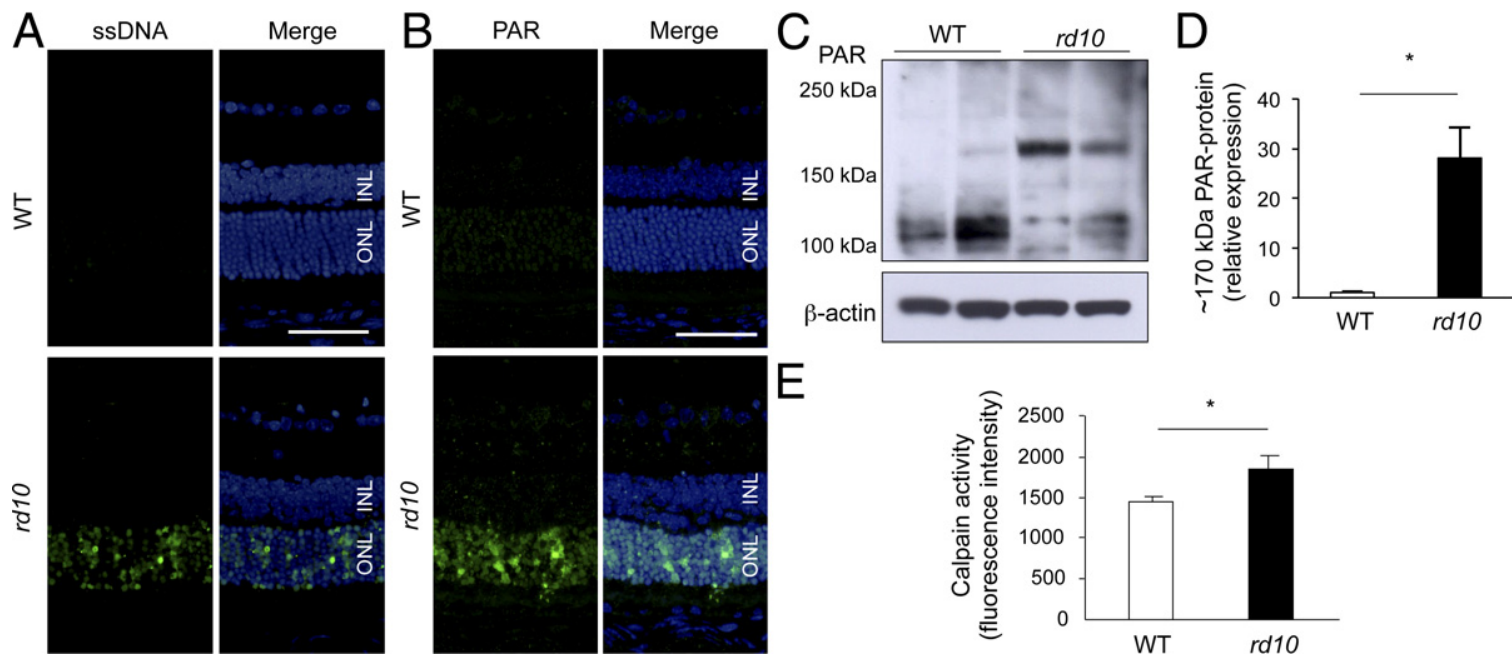

Figure 3. Formation of SSBs and activation of PARP and calpain pathways in $r d 10$ mice. A: Immunofluorescence for single-strand DNA (ssDNA; green) and DAPI (blue) in p21 WT and $r d 10$ mice. INL, inner nuclear layer; ONL, outer nuclear laver. B: Immunofluorescence for PAR (green) and DAPI (blue) in p21 WT and $r d 10$ mice. C and D: Western blot analysis for the PAR-protein $(\mathbf{C})$ and quantitative densitometric analysis of the $\sim 170-\mathrm{kDa}$ PAR-protein (D) in retinal lysates of p21 WT and $r d 10$ mice $(n=6$ each). The amount of the $\sim 170-\mathrm{kDa}$ PAR-protein increased 28 -fold in the $r d 10$ retina compared with the WT retina. Lane-loading differences were normalized by the level of $\beta$-actin. ${ }^{*} P=0.0039$. E: Calpain activity in the retinal lysates of p21 WT and $r d 10$ mice was measured by a substrate-based assay. The $r d 10$ retina showed higher calpain activity than the WT retina. ${ }^{*} P=0.0374$. Scale bars: $50 \mu \mathrm{m}(\mathbf{A}$ and $\mathbf{B})$.

inhibitors reduced PAR overproduction and calpain activity, respectively, in rd10 mice (see Supplemental Figure S3 at http://ajp.amjpathol.org). At p26, the PARP inhibitor significantly decreased the photoreceptor cell loss compared with vehicle treatment $(P<0.01)$, whereas the calpain inhibitor showed no significant protective effect (Figure 4, A and B). Cotreatment with the PARP and calpain inhibitors provided no additional protective effect compared with the PARP inhibitor alone (Figure 4, A and B). These data suggest that PARP is a predominant pathway in the mediation of photoreceptor cell death in rd10 mice.

\section{MTH1 Regulates SSB Formation, PAR Production, and AIF Nuclear Translocation in rd10 Mice}

Finally, we addressed whether DNA oxidative damage acts as an upstream event of SSB formation and PARP activation during retinal degeneration. The retina sections of rd10; hMTH1- $\mathrm{Tg}^{+/+}$mice showed significantly less staining for single-strand DNA than retina sections of rd10; $h M M T H 1-\mathrm{Tg}^{-/-}$ mice (Figure 5A). PAR expression in the ONL was also substantially reduced in rd10; $h \mathrm{MTH} 1-\mathrm{Tg}^{+/+}$mice (Figure 5B). AlF is a flavoprotein in the mitochondrial intermembrane space, and its nuclear translocation induces caspase-independent cell death. ${ }^{34}$ Because PAR polymer is a potent AIF-releasing factor, ${ }^{35}$ we analyzed the cellular localization of AIF by immunofluorescence. In rd10; hMTH1$\mathrm{Tg}^{-/-}$mice, AlF nuclear translocation was observed in most of the TUNEL-positive cells. In contrast, hMTH1 overexpression substantially reduced AIF nuclear translocation (Figure $5 C)$. Together, these data indicate that DNA oxidation in photoreceptor cells, which mainly accumulates in their nuclei, induces SSB formation, PARP activation, AIF nuclear translocation, and the subsequent photoreceptor cell death in $r d 10$ mice.

\section{Discussion}

Oxidative stress influences diverse biological processes, including cell homeostasis, mutagenesis, and death, as
A

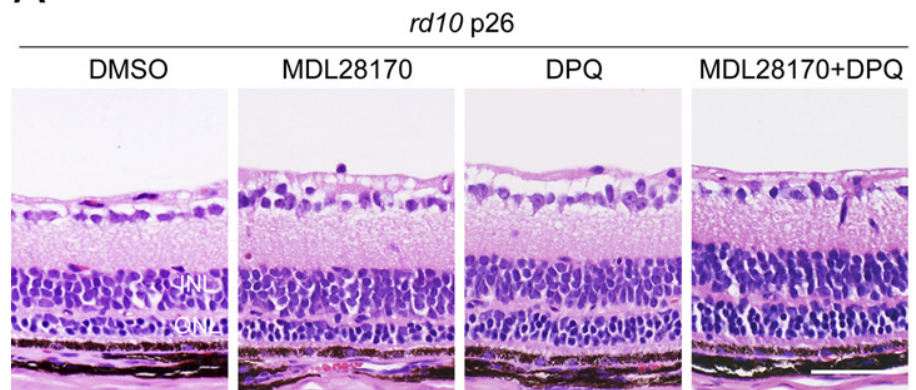

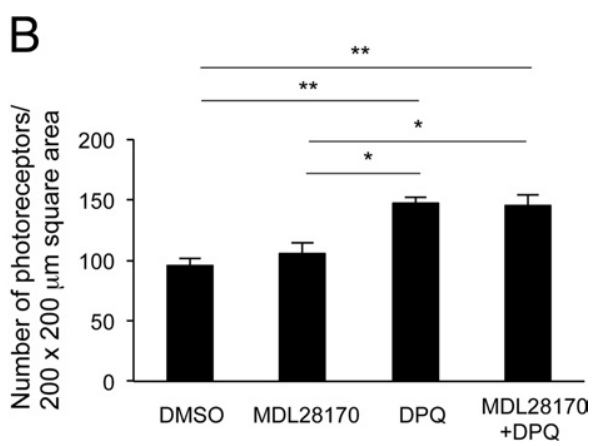

Figure 4. PARP inhibition attenuates photoreceptor cell loss in $r d 10$ mice. A: Histologic findings of the retina in p26 $r d 10$ mice receiving a daily intraperitoneal injection of dimethyl sulfoxide (DMSO), calpain inhibitor MDL28170, PARP inhibitor DPQ, or MDL28170 plus DPQ from p19 to p26. Scale bar $=50 \mu \mathrm{m}$. B: Quantification of cells in the outer nuclear layer (ONL). Treatment with DPQ attenuated photoreceptor cell loss compared with DMSO treatment, whereas MDL28170 provided no significant protective effect $\left(n=8\right.$ each). INL, inner nuclear layer. ${ }^{*} P<0.05,{ }^{* * *} P<0.01$. 


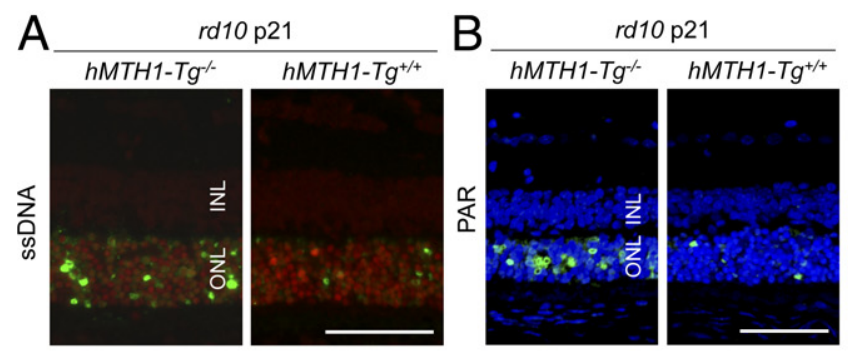

Figure 5. MTH1 overexpression suppresses SSB formation, PAR production, and AIF nuclear translocation in $r d 10$ mice. A: Immunofluorescence for singlestrand DNA (ssDNA; green) and propidium iodide (red) in p21 rd10; hMTH1 $\mathrm{Tg}^{-/-}$or $\mathrm{rd10}, \mathrm{hMTH1}-\mathrm{Tg}^{+/+}$mice. INL, inner nuclear layer, IS, inner segment; ONL, outer nuclear laver. B: Immunofluorescence for PAR (green) and DAPI (blue) in p21 rd10; $\mathrm{hMTH1}-\mathrm{Tg}^{-/-}$or $\mathrm{rd} 10 ; \mathrm{hMTH1}-\mathrm{Tg}^{+/+}$mice. C: Immunofluorescence for AIF (red) and TUNEL (green) in p21 WT, rd10; $\mathrm{hMTH1}^{-\mathrm{Tg}^{-1-}}$ or rd10; $h$ MTH1 $-\mathrm{Tg}^{+/+}$mice. Scale bars: $50 \mu \mathrm{m}(\mathbf{A}$ and $\mathbf{B}) ; 20 \mu \mathrm{m}(\mathbf{C})$.

well as the pathogenesis of many diseases that range from cancer to neurodegeneration. Although accumulating evidence indicates that oxidative stress is involved in $\mathrm{RP},{ }^{2,3}$ the underlying mechanisms by which oxidative stress causes photoreceptor cell death remain largely unknown. In the present study, we show that oxidative DNA damage accumulates in photoreceptor cells, especially in the nuclei, from an early phase of retinal degeneration in two models of RP. Furthermore, we found that overexpression of $\mathrm{hMTH} 1$, an enzyme that hydrolyzes oxidized purine nucleoside triphosphatase in the nucleotide pool, attenuates photoreceptor cell death in rd10 mice, in addition to suppressing SSB formation, PARP activation, and AIF nuclear translocation. These data indicate that oxidation of purine nucleotides such as 8-oxodGTP and their accumulation in nuclear DNA are important processes that initiate cell-death signaling in $r d 10$ mice. However, our results show that transgenic hMTH1 overexpression delays, but does not prevent, photoreceptor cell loss in rd10 mice, suggesting that other oxidative insults such as oxidation of proteins and lipids may also contribute to photoreceptor degeneration.

In this study, we used two genetically different animal models of RP. Mice with spontaneous mutations in the $P d e 6 \beta$ gene are one of the most studied animal models of $\mathrm{RP}$, and mutations in this gene have been found in human patients with autosomal recessive RP. ${ }^{1,36}$ Loss-of-function mutations in this gene lead to disruption of the rod phototransduction cascade and retinal degeneration. $\mathrm{RCS}$ rats are another clinically relevant model of RP that carries a mutation in the receptor tyrosine kinase Mertk. ${ }^{22,23}$ Loss of Mertk function results in defective phagocytosis of the photoreceptor outer segment by the retinal pigment epithelium. Although the function of $P d e 6 \beta$ and Mertk is different, the dying rod photoreceptor cells show similar apoptotic features in these animals, along with the activation of common cell death pathways such as the nuclear translocation of AIF. 18,30,37 These findings suggest that different mechanisms might converge onto a final common death pathway, at least in part. Inspired by our previous in vitro work showing that oxidative DNA damage is crucial for AIF nuclear translo-

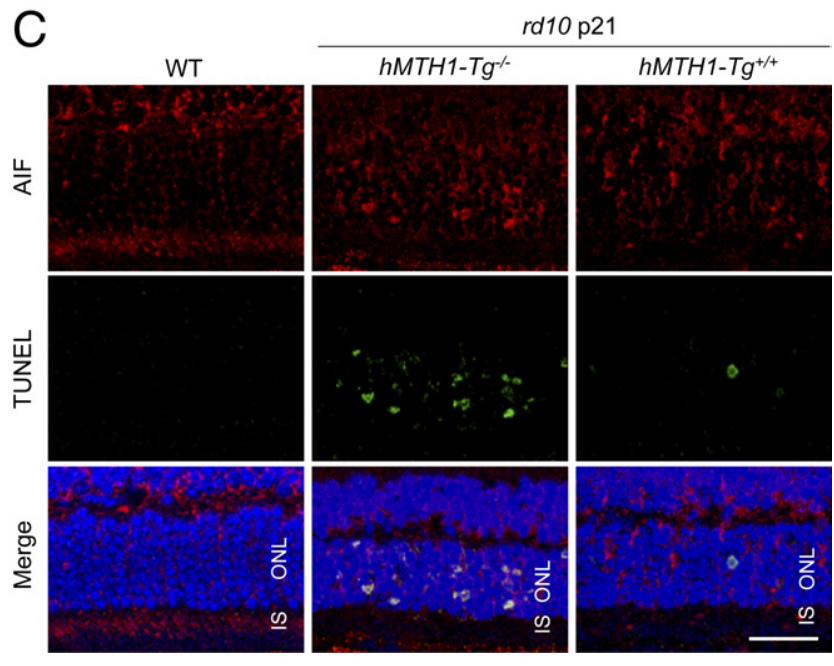

cation and cell death, ${ }^{27}$ we investigated these pathways and found that the photoreceptor cells in both $\mathrm{rd10}$ mice and RCS rats exhibit enhanced 8-oxo-dG accumulation. In addition, we showed that vitreous samples from patients with RP contain higher levels of 8-oxo-dG compared with samples from patients without retinal degeneration. Although genetic mutations in patients with RP were not identified in this study, the variable inheritance patterns and clinical courses suggest that increased oxidation of nucleic acids may be associated with heterogeneous conditions of RP. Further comprehensive studies to investigate the correlation among genes, retinal degeneration, and oxidative DNA damage will be important for a better understanding of the mechanisms of RP.

Accumulation of 8-oxo-dG has been shown to occur mainly in the mitochondrial DNA of degenerating neurons in Alzheimer's disease, Parkinson's disease, and amyotrophic lateral sclerosis. ${ }^{8,9,38}$ In contrast, we here found that 8-oxo-dG is accumulated mainly in the photoreceptor nuclei in two animal models of RP. The reasons for this discrepancy are currently unclear. However, because photoreceptor cells consume a large amount of ATP for phototransduction, ${ }^{39}$ the mitochondria of photoreceptor cells may have an elaborate defense system against reactive oxygen species produced during oxidative phosphorylation. ${ }^{39} \mathrm{NADPH}$ oxidase complexes that produce superoxide are an alternative source of reactive oxygen species. ${ }^{40}$ We observed that the expression levels of NADPH oxidase 2 are increased in the retina of rd10 mice compared with WT mice (Y. Murakami and Y. Ikeda, unpublished observations). Because rod photoreceptor-specific knockout of Rac1, a component of NADPH oxidase, protects rods against cell death after light injury, ${ }^{41}$ enhanced NADPH oxidase activity in photoreceptor cells may contribute to retinal oxidative damage and degeneration under pathologic conditions. The upstream molecular mechanisms by which the nuclear DNA of photoreceptor cells are oxidized in rd10 mice and RCS rats warrant further investigation.

Oxidative DNA damage can derive from direct oxidation of bases in DNA or from incorporation of oxidized nucleotides generated in the nucleotide pools. It has 
been shown that in vitro exposure of free guanine nucleotides to reactive oxygen species generates eight to nine times more 8-oxo-dG residues than comparable exposure of DNA. ${ }^{42}$ MTH1 is a main enzyme for the sanitization of nucleotide pools. ${ }^{43}$ In this study, we showed that hMTH1 overexpression suppresses 8-oxo-dG accumulation in the photoreceptor nuclei of $r d 10$ mice. These data suggest that a substantial fraction of oxidative nuclear DNA damage in rd10 mouse retina is derived from the oxidized nucleotide pool. We previously reported that the incorporation of oxidized bases into nuclear DNA occurs during DNA replication in proliferating cells. ${ }^{27}$ However, it is less likely that the oxidative nuclear DNA lesions detected in photoreceptors are incorporated from the oxidized nucleotide pool during cell division in retinal development, because the expression of rod-specific genes, including Pde6 $\beta$, starts after terminal mitosis. ${ }^{44}$ Recent accumulating evidence indicates that postmitotic neurons re-enter the cell cycle in response to DNA damage, to activate the DNA repair system. ${ }^{45}$ Thus, degenerating photoreceptors may incorporate oxidized dNTPs in the process of repair replication, which in turn could lead to exacerbation of oxidative nuclear DNA damage, and finally result in accumulation of SSBs through further DNA repair processes.

PARP is an enzyme that is activated by SSBs in nuclear DNA and involved in DNA repair. ${ }^{46}$ However, excessive activation of PARP leads to consumption of nicotinamide adenine dinucleotide and production of PAR polymer, a potent AIF-releasing factor. ${ }^{35}$ We previously reported that 8-oxoG accumulated in nuclear DNA causes PARP-dependent cell death in cultured fibroblasts. ${ }^{27}$ Consistent with those findings, we here showed that photoreceptor cells of rd10 mice, in which 8-oxo-dG is accumulated mainly in the nuclei, are associated with strong activation of the PARP pathway and treatment with the PARP inhibitor delays photoreceptor cell death. Furthermore, we found that transgenic hMTH1 overexpression effectively suppresses PAR overproduction and AIF nuclear translocation in rd10 mice. These findings suggest that DNA damage derived from the oxidized nucleotide pool is a mediator of PARP-AIF signaling in rd10 mice. Although the PARP pathway has been shown to participate in retinal cell death in the developmental phase, ${ }^{47}$ we observed that hMTH1 overexpression does not affect cell death during retinal development (see Supplemental Figure S4 at http://ajp.amjpathol.org), suggesting that cell death in the postnatal retina and in rd10 mouse retina may be induced by different mechanisms, at least in part.

Although clinical trials of nutritional interventions, including vitamin A and docosahexaenotic acid, have been conducted in patients with RP, the results did not show clear efficacy in slowing the progression of vision loss, ${ }^{1}$ and these diseases remain a main cause of adult blindness. Thus, identification of the mechanisms that underlie the pathogenesis of RP is critical to developing new treatment strategies. In this study, we found that oxidation of nucleic acids is associated with animal models and human vitreous samples of RP and that overexpression of hMTH1 with oxidized purine nucleoside triphosphatase activity attenuates photoreceptor cell death in rd10 mice.
These findings indicate that oxidative DNA damage contributes to photoreceptor cell death in rd10 mice and suggest that DNA oxidation may be a therapeutic target for preventing vision loss in individuals with RP.

\section{Acknowledgments}

We thank Hiroshi Fujii and Chie Arimatsu for their assistance with the experiments. KN International (Chicago, $\mathrm{IL})$ provided language assistance.

\section{References}

1. Hartong DT, Berson EL, Dryja TP: Retinitis pigmentosa. Lancet 2006, 368:1795-1809

2. Komeima K, Rogers BS, Lu L, Campochiaro PA: Antioxidants reduce cone cell death in a model of retinitis pigmentosa. Proc Natl Acad Sci U S A 2006, 103:11300-11305

3. Komeima K, Rogers BS, Campochiaro PA: Antioxidants slow photoreceptor cell death in mouse models of retinitis pigmentosa. J Cell Physiol 2007, 213:809-815

4. Chong ZZ, Li F, Maiese K: Oxidative stress in the brain: novel cellular targets that govern survival during neurodegenerative disease. Prog Neurobiol 2005, 75:207-246

5. Mackey AM, Sanvicens N, Groeger G, Doonan F, Wallace D, Cotter TG: Redox survival signalling in retina-derived $661 \mathrm{~W}$ cells. Cell Death Differ 2008, 15:1291-1303

6. Chen J, Jin K, Chen M, Pei W, Kawaguchi K, Greenberg DA, Simon RP: Early detection of DNA strand breaks in the brain after transient focal ischemia: implications for the role of DNA damage in apoptosis and neuronal cell death. J Neurochem 1997, 69:232-245

7. Guillet M, Boiteux S: Endogenous DNA abasic sites cause cell death in the absence of Apn1. Apn2 and Rad1/Rad10 in Saccharomyces cerevisiae, EMBO J 2002, 21:2833-2841

8. Zhang J, Perry G, Smith MA, Robertson D, Olson SJ, Graham DG, Montine TJ: Parkinson's disease is associated with oxidative damage to cytoplasmic DNA and RNA in substantia nigra neurons. Am J Pathol 1999, 154:1423-1429

9. Nunomura A, Perry G, Aliev G, Hirai K, Takeda A, Balraj EK, Jones PK, Ghanbari H, Wataya T, Shimohama S, Chiba S, Atwood CS, Petersen RB, Smith MA: Oxidative damage is the earliest event in Alzheimer disease. J Neuropathol Exp Neurol 2001, 60:759-767

10. Boiteux S, Radicella JP: The human OGG1 gene: structure, functions, and its implication in the process of carcinogenesis. Arch Biochem Biophys 2000, 377:1-8

11. Maki H, Sekiguchi M: MutT protein specifically hydrolyses a potent mutagenic substrate for DNA synthesis. Nature 1992, 355:273-275

12. Sakumi K, Furuichi M, Tsuzuki T, Kakuma T, Kawabata S, Maki H, Sekiguchi M: Cloning and expression of cDNA for a human enzyme that hydrolyzes 8-oxo-dGTP, a mutagenic substrate for DNA synthesis. J Biol Chem 1993, 268:23524-23530

13. Yamaguchi H, Kajitani K, Dan Y, Furuichi M, Ohno M, Sakumi K, Kang D, Nakabeppu Y: MTH1, an oxidized purine nucleoside triphosphatase, protects the dopamine neurons from oxidative damage in nucleic acids caused by 1-methyl-4-phenyl-1,2,3,6-tetrahydropyridine. Cell Death Differ 2006, 13:551-563

14. De Luca G, Russo MT, Degan P, Tiveron C, Zijno A, Meccia E, Ventura I, Mattei E, Nakabeppu Y, Crescenzi M, Pepponi R, Pezzola A, Popoli P, Bignami M: A role for oxidized DNA precursors in Huntington's disease-like striatal neurodegeneration, PLoS Genet 2008, 4:e1000266

15. Murakami T, Akimoto $M$, Ooto $S$, Suzuki $T$, Ikeda $H$, Kawagoe $N$, Takahashi M, Yoshimura N: Association between abnormal autofluorescence and photoreceptor disorganization in retinitis pigmentosa. Am J Ophthalmol 2008, 145:687-694

16. Hayakawa M, Fujiki K, Kanai A, et al: Multicenter genetic study of retinitis pigmentosa in Japan, I: genetic heterogeneity in typical retinitis pigmentosa. Jpn J Ophthalmol 1997, 41:1-6

17. Tsuruya K, Furuichi M, Tominaga $Y$, Shinozaki M, Tokumoto M, Yoshimitsu T, Fukuda K, Kanai H, Hirakata H, lida M, Nakabeppu Y: 
Accumulation of 8-oxoguanine in the cellular DNA and the alteration of the OGG1 expression during ischemia-reperfusion injury in the rat kidney. DNA Repair (Amst) 2003, 2:211-229

18. Murakami $Y$, Ikeda $Y$, Yonemitsu $Y$, Onimaru M, Nakagawa K, Kohno R, Miyazaki M, Hisatomi T, Nakamura M, Yabe T, Hasegawa M, Ishibashi T, Sueishi K: Inhibition of nuclear translocation of apoptosisinducing factor is an essential mechanism of the neuroprotective activity of pigment epithelium-derived factor in a rat model of retinal degeneration. Am J Pathol 2008, 173:1326-1338

19. Chang B, Hawes NL, Hurd RE, Davisson MT, Nusinowitz S, Heckenlively JR: Retinal degeneration mutants in the mouse. Vision Res 2002, 42:517-525

20. Pang JJ, Boye SL, Kumar A, Dinculescu A, Deng W, Li J, Li Q, Rani A, Foster TC, Chang B, Hawes NL, Boatright JH, Hauswirth WW: AAV-mediated gene therapy for retinal degeneration in the rd10 mouse containing a recessive PDEbeta mutation. Invest Ophthalmol Vis Sci 2008, 49:4278-4283

21. Kasai H, Nishimura S: Hydroxylation of deoxyguanosine at the C-8 position by ascorbic acid and other reducing agents. Nucleic Acids Res 1984, 12:2137-2145

22. D'Cruz PM, Yasumura D, Weir J, Matthes MT, Abderrahim H, LaVail MM, Vollrath D: Mutation of the receptor tyrosine kinase gene Mertk in the retinal dystrophic RCS rat. Hum Mol Genet 2000, 9:645-651

23. Gal A, Li Y, Thompson DA, Weir J, Orth U, Jacobson SG, ApfelstedtSylla E, Vollrath D: Mutations in MERTK, the human orthologue of the $\mathrm{RCS}$ rat retinal dystrophy gene, cause retinitis pigmentosa. Nat Genet 2000, 26:270-271

24. Shigenaga MK, Gimeno CJ, Ames BN: Urinary 8-hydroxy-2'-deoxyguanosine as a biological marker of in vivo oxidative DNA damage. Proc Natl Acad Sci U S A 1989, 86:9697-9701

25. Sato S, Mizuno Y, Hattori N: Urinary 8-hydroxydeoxyguanosine levels as a biomarker for progression of Parkinson disease. Neurology 2005, 64:1081-1083

26. Shiihara T, Kato M, Ichiyama T, Takahashi Y, Tanuma N, Miyata R, Hayasaka K: Acute encephalopathy with refractory status epilepticus: bilateral mesial temporal and claustral lesions, associated with a peripheral marker of oxidative DNA damage. J Neurol Sci 2006, 250:159-161

27. Oka S, Ohno M, Tsuchimoto D, Sakumi K, Furuichi M, Nakabeppu Y: Two distinct pathways of cell death triggered by oxidative damage to nuclear and mitochondrial DNAs. EMBO J 2008, 27:421-432

28. Yu SW, Wang $H$, Poitras MF, Coombs C, Bowers WJ, Federoff HJ, Poirier GG, Dawson TM, Dawson VL: Mediation of poly(ADP-ribose) polymerase-1-dependent cell death by apoptosis-inducing factor. Science 2002, 297:259-263

29. Paquet-Durand F, Silva J, Talukdar T, Johnson LE, Azadi S, van Veen T, Ueffing M, Hauck SM, Ekstrom PA: Excessive activation of poly(ADP-ribose) polymerase contributes to inherited photoreceptor degeneration in the retinal degeneration 1 mouse. J Neurosci 2007 , 27:10311-10319

30. Sanges D, Comitato A, Tammaro R, Marigo V: Apoptosis in retinal degeneration involves cross-talk between apoptosis-inducing factor (AIF) and caspase-12 and is blocked by calpain inhibitors. Proc Natl Acad Sci U S A 2006, 103:17366-17371

31. Mizukoshi S, Nakazawa M, Sato K, Ozaki T, Metoki T, Ishiguro S: Activation of mitochondrial calpain and release of apoptosis-inducing factor from mitochondria in RCS rat retinal degeneration. Exp Eye Res 2010, 91:353-361

32. Takahashi K, Pieper AA, Croul SE, Zhang J, Snyder SH, Greenberg $\mathrm{JH}$ : Post-treatment with an inhibitor of poly(ADP-ribose) polymerase attenuates cerebral damage in focal ischemia. Brain Res 1999 , 829:46-54

33. Kawamura M, Nakajima W, Ishida A, Ohmura A, Miura S, Takada G: Calpain inhibitor MDL 28170 protects hypoxic-ischemic brain injury in neonatal rats by inhibition of both apoptosis and necrosis. Brain Res 2005, 1037:59-69

34. Susin SA, Lorenzo HK, Zamzami N, Marzo I, Snow BE, Brothers GM, Mangion J, Jacotot E, Costantini P, Loeffler M, Larochette N, Goodlett DR, Aebersold R, Siderovski DP, Penninger JM, Kroemer G: Molecular characterization of mitochondrial apoptosis-inducing factor. Nature 1999, 397:441-446

35. Yu SW, Andrabi SA, vWang H, Kim NS, Poirier GG, Dawson TM, Dawson VL: Apoptosis-inducing factor mediates poly(ADP-ribose) (PAR) polymer-induced cell death, Proc Natl Acad Sci U S A 2006, 103:18314-18319

36. McLaughlin ME, Sandberg MA, Berson EL, Dryja TP: Recessive mutations in the gene encoding the beta-subunit of rod phosphodiesterase in patients with retinitis pigmentosa. Nat Genet 1993, 4:130134

37. Chang GQ, Hao Y, Wong F: Apoptosis: final common pathway of photoreceptor death in rd, rds, and rhodopsin mutant mice. Neuron 1993, 11:595-605

38. Kikuchi H, Furuta A, Nishioka K, Suzuki SO, Nakabeppu Y, Iwaki T: Impairment of mitochondrial DNA repair enzymes against accumulation of 8-oxo-guanine in the spinal motor neurons of amyotrophic lateral sclerosis. Acta Neuropathol 2002, 103:408-414

39. Laughlin SB, de Ruyter van Steveninck RR, Anderson JC: The metabolic cost of neural information. Nat Neurosci 1998, 1:36-41

40. Takeya R, Sumimoto H: Molecular mechanism for activation of superoxide-producing NADPH oxidases. Mol Cells 2003, 16:271-277

41. Haruta M, Bush RA, Kjellstrom S, Vijayasarathy C, Zeng Y, Le YZ, Sieving PA: Depleting Rac1 in mouse rod photoreceptors protects them from photo-oxidative stress without affecting their structure or function. Proc Natl Acad Sci U S A 2009, 106:9397-9402

42. Kamiya $\mathrm{H}$, Kasai $\mathrm{H}$ : Formation of 2-hydroxydeoxyadenosine triphosphate, an oxidatively damaged nucleotide, and its incorporation by DNA polymerases. Steady-state kinetics of the incorporation, J Biol Chem 1995, 270:19446-19450

43. Nakabeppu Y, Oka S, Sheng Z, Tsuchimoto D, Sakumi K: Programmed cell death triggered by nucleotide pool damage and its prevention by MutT homolog-1 (MTH1) with oxidized purine nucleoside triphosphatase. Mutat Res 2010, 703:51-58

44. Akimoto M, Cheng H, Zhu D, Brzezinski JA, Khanna R, Filippova E, Oh EC, Jing Y, Linares JL, Brooks M, Zareparsi S, Mears AJ, Hero A, Glaser vT, Swaroop A: Targeting of GFP to newborn rods by $\mathrm{Nrl}$ promoter and temporal expression profiling of flow-sorted photoreceptors, Proc Natl Acad Sci U S A 2006, 103:3890-3895

45. Kruman II, Wersto RP, Cardozo-Pelaez F, Smilenov L, Chan SL, Chrest FJ, Emokpae R Jr, Gorospe M, Mattson MP: Cell cycle activation linked to neuronal cell death initiated by DNA damage. Neuron 2004, 41:549-561

46. Kauppinen TM, Swanson RA: The role of poly(ADP-ribose) polymerase-1 in CNS disease. Neuroscience 2007, 145:1267-1272

47. Martin-Oliva D, Ferrer-Martin RM, Santos AM, Carrasco MC, Sierra A, Marin-Teva JL, Calvente R, Navascues J, Cuadros MA: Simultaneous cell death and upregulation of poly(ADP-ribose) polymerase-1 expression in early postnatal mouse retina. Invest Ophthalmol Vis Sci 2011, 52:7445-7454 